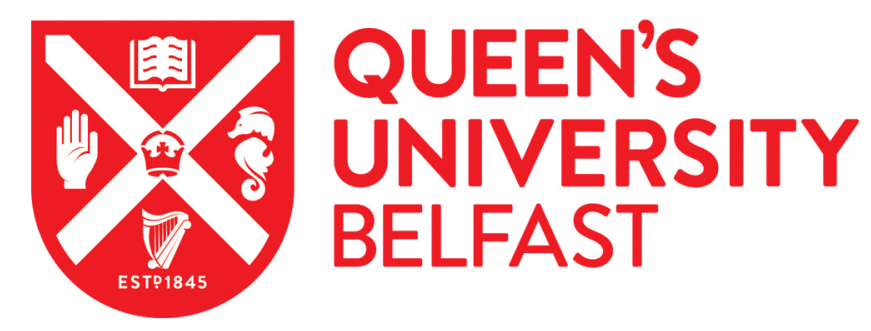

\title{
eHealth interventions for reducing cardiovascular disease risk in men: A systematic review and meta-analysis
}

McMahon, J., Thompson, D. R., Pascoe, M. C., Brazil, K., \& Ski, C. F. (2021). eHealth interventions for reducing cardiovascular disease risk in men: A systematic review and meta-analysis. Preventive Medicine, 145, [106402]. https://doi.org/10.1016/j.ypmed.2020.106402

Published in:

Preventive Medicine

Document Version:

Peer reviewed version

Queen's University Belfast - Research Portal:

Link to publication record in Queen's University Belfast Research Portal

\section{Publisher rights}

Copyright 2020 Elsevier.

This manuscript is distributed under a Creative Commons Attribution-NonCommercial-NoDerivs License

(https://creativecommons.org/licenses/by-nc-nd/4.0/), which permits distribution and reproduction for non-commercial purposes, provided the author and source are cited.

\section{General rights}

Copyright for the publications made accessible via the Queen's University Belfast Research Portal is retained by the author(s) and / or other copyright owners and it is a condition of accessing these publications that users recognise and abide by the legal requirements associated with these rights.

Take down policy

The Research Portal is Queen's institutional repository that provides access to Queen's research output. Every effort has been made to ensure that content in the Research Portal does not infringe any person's rights, or applicable UK laws. If you discover content in the Research Portal that you believe breaches copyright or violates any law, please contact openaccess@qub.ac.uk. 


\section{Review}

eHealth interventions for reducing cardiovascular disease risk in men: a systematic review and meta-analysis

James McMahon ${ }^{a}$, David R Thompson ${ }^{\mathrm{a}}$, Michaela C Pascoe ${ }^{\mathrm{b}}$, Kevin Brazila ${ }^{\mathrm{a}}$, Chantal F ${ }^{*} \mathbf{S k i}^{\mathrm{a}, \mathrm{c}}$

a School of Nursing and Midwifery, Queen's University Belfast, Belfast, UK

${ }^{b}$ Institute for Health \& Sport, Victoria University, Melbourne, Australia

${ }^{c}$ Integrated Care Academy, University of Suffolk, Ipswich, UK

Keywords: eHealth interventions; Cardiovascular risk; Men; Systematic review; Meta-analysis

*Corresponding author

Email address: C.Ski@qub.ac.uk

Word count: 248 (abstract); 3998 (main text) 


\section{ABSTRACT}

Men remain at a higher risk of developing cardiovascular disease (CVD) than women and behavioral risk factor modification is an important preventive measure. However, engaging men in behavior change interventions is challenging. Although men often indicate a preference for gender-specific information and support, this rarely occurs. eHealth interventions have the potential to address this gap, though their effectiveness for reducing CVD risk in men is unclear. Therefore, the aim of this systematic review and meta-analysis was to evaluate the effectiveness of eHealth interventions for reducing CVD risk in men. A search of published randomised controlled trials with no date restrictions up to July 2020 was conducted to identify those targeting at least two major CVD risk factors. Nine trials were identified and reviewed. Study quality ranged from low to unclear, with one trial at a high risk of bias. Compared to those in a control group or receiving printed materials, participants randomised to an eHealth intervention had statistically significant improvements in BMI $(Z=-2.75, p=0.01)$, body weight $(Z=-3.25, p=0.01)$, waist circumference $(Z=-2.30$, $p=0.02)$ and systolic $(Z=-3.57, p=0.01)$ and diastolic $(Z=-3.56, p=0.01)$ blood pressure. Though less evident, there were also improvements in physical activity and diet in favour of the intervention group. This review suggests that eHealth interventions can reduce CVD risk in adult men through behavior change. However, we were unable to determine the association between intervention characteristics and outcomes. Also, overall, participant adherence to the intervention was poor. Both of these issues should be considered in future studies. 


\section{Introduction}

Cardiovascular disease (CVD) is the leading cause of death and disability, with men at an increased risk compared to women and at an earlier age (Virani et al., 2020). The higher risk can partly be explained by male lifestyle choices, such as poor dietary habits (Vari et al., 2016; White et al., 2011) and a higher consumption of alcohol (Moinuddin and Goel, 2016; White et al., 2011), as well as poor health seeking behaviors compared to females (White et al., 2011; Yousaf et al., 2015). Behavioral risk factor modification of physical inactivity, poor diet, smoking and alcohol consumption is key in the prevention of CVD (Turco et al., 2018; World Health Organization, 2017). However, engaging men in behavior change interventions aimed at reducing CVD is challenging and uptake is low. Although men express a preference for interventions to be targeted specifically at them (Drew et al., 2020; Gavarkovs et al., 2016; Young et al., 2012), this rarely occurs. In a review of 244 weight loss studies, only five were targeted specifically at men (Pagoto et al., 2012). Similarly, in two systematic reviews, one of 58 studies aimed at increasing physical activity (Malik et al., 2014), and the other of 51 studies aimed at reducing sedentary behaviors (Martin et al., 2015), only two studies in each comprised a male only sample.

An additional challenge lies in the recruitment of men to participate in such studies. A scoping review of 25 chronic disease prevention and management studies (Gavarkovs et al., 2016) identified several barriers to engaging men in lifestyle-based interventions, including work commitments, poor health seeking behaviors and perceptions that the interventions were 'inherently feminine'. Men appear to accrue greater benefits from a gender-tailored intervention that takes into consideration the method of engagement, how the information is presented and its mode of delivery (Duncan et al., 2012; Gavarkovs et al., 2016). To improve engagement, uptake and adherence by men, interventions need to be tailored to males using gender-specific information, offer a preferred mode of delivery and include male only participants (Bottorff et al., 2015; Gavarkovs et al., 2016). eHealth interventions show much potential to meet these needs via easily accessible and individually tailored content (De Vries and Brug, 1999; Muellmann et al., 2016). 
Systematic reviews and meta-analyses have demonstrated the positive impact of eHealth interventions on common CVD risk factors, such as promoting physical activity (Davies et al., 2012; Jahangiry et al., 2017; Kroeze et al., 2006; Norman et al., 2007) and healthy dietary behaviors (Kroeze et al., 2006; Norman et al., 2007) among adults, yet there is a dearth of evidence pertaining to male only samples. Exceptions are male-only weight loss programs which may effectively engage and assist men with weight loss (Young et al., 2012) and male-only lifestyle interventions which improve men's mental health in some circumstances (Drew et al., 2020), though the authors of these reviews note that more high quality studies are needed. The aim of this study was to systematically review and metaanalyse published randomised controlled trials to identify the effectiveness of eHealth interventions for reducing CVD risk in men.

\section{Methods}

This review was conducted in accordance with the Preferred Reporting Items for Systematic Reviews and Meta-Analyses (PRISMA) statement (Moher et al., 2009) and principles laid out by the Cochrane Handbook for Systematic Reviews of Interventions (Higgins et al., 2019). A protocol for this systematic review is located on the PROSPERO international database (identification number: CRD42019137841).

\section{Search strategy}

We conducted a search of published randomised controlled trials (RCTs) of eHealth interventions for reducing CVD risk in men through the Cochrane Central register of Controlled Trials (CENTRAL), Medline, CINAHL Plus, PsycINFO and SCOPUS databases, initially in May 2019 then updated in July 2020; no restriction was placed on year of publication. Reference lists of relevant systematic reviews and meta-analyses were searched manually for additional trials not found during the search. Articles published only as an abstract or a conference presentation or in a language other than English were excluded.

\section{Study eligibility}

Eligible studies were RCTs that used eHealth interventions targeting a minimum of two major CVD-related risk factors (e.g. weight, physical activity, diet) in males aged over 17 years. 
eHealth is defined as the use of technology (in this instance mobile phones, computers, laptops, tablets) to improve health, wellbeing and healthcare (van Gemert-Pijnen et al., 2018).

\section{Study selection process}

All studies identified through the search strategy were imported into Covidence, a software platform that streamlines the production of systematic reviews. Once duplicates were removed, remaining titles and abstracts were screened independently by three reviewers (JM, DRT and KB). Remaining studies then underwent full text review by two reviewers (JM and $\mathrm{KB})$. Any disagreements during screening and full text review were resolved through a fourth reviewer (CFS).

\section{Data extraction}

Data extraction was conducted independently by two reviewers (JM and $\mathrm{KB}$ ) using a predesigned data extraction form. Any disagreements were then discussed and settled by a third reviewer (CFS). The following data were extracted: country undertaken, aims, setting, participant and intervention characteristics, study methodology, and outcomes. Means $(M)$, standard deviations $(S D)$ and sample sizes $(n)$ were also extracted.

\section{Data analysis and synthesis}

Meta-analysis was undertaken using Comprehensive Meta-Analysis Version 3 (CMA Version3) software. The analysis compared the effect of the intervention with the control or comparison group on body mass index $\left(\mathrm{kg} / \mathrm{m}^{2}\right)$, body weight $(\mathrm{kg})$, waist circumference $(\mathrm{cm})$, systolic blood pressure $(\mathrm{mmHg})$ and diastolic blood pressure $(\mathrm{mmHg})$. A funnel plot was used to examine publication bias. Sensitivity analyses were performed using 'one-study-removed' method; results of this are only presented in text when removal of a study affected the outcome. A random-effects model was used in all analyses, weighting the studies based on the sample size/standard error. In cases when pre-post correlations were not reported in the published papers, we used a 0.5 correlation.

For the meta-analysis we report the mean difference. Hedges' $G(g)$ form of the SMD was used. Results using completers only were used when intention-to-treat (ITT) results were not reported. We report the confidence interval $(\mathrm{Cl})$, the $Z$-value, $p$-value and $I^{2}$ statistic 
(Borenstein et al., 2009). For those outcomes not included in the meta-analysis, we provide a narrative synthesis.

\section{Quality assessment}

The quality of the included trials was assessed using the Cochrane risk of bias tool (Version 1) (Higgins et al., 2011) concurrently by two reviewers (JM and KB). The following sources of bias were assessed: sequence generation, allocation concealment, blinding of participants and personnel, blinding of outcome assessor, incomplete outcome data and selective outcome reporting. Risk of bias across all domains was assessed using the classifications recommended by Cochrane (Higgins et al., 2019) (Table 1). A summary risk of bias for each study was also created using these criteria. Heterogeneity between studies was assessed using $l^{2}$ in the quality assessment.

Table 1. Risk of bias classification criteria (Higgins et al., 2019)

- Low risk of bias: All domains judged to be at a low risk of bias

- Some concern (unclear): Judged to raise some concerns (unclear) in at least one domain

- High risk of bias: A minimum of one domain judged to be at a high risk of bias Or

- $\quad$ Multiple domains judged to be unclear and therefore lowering the confidence in the study

\section{Results}

\section{Study selection}

A total of 3168 records were retrieved through the database searches with 2014 titles and abstracts screened for eligibility once duplicates were removed. Articles that appeared appropriate for this review following title and abstract screening $(n=66)$ were assessed via full text screening for their eligibility. Nine trials were assessed as eligible for inclusion in this review. A PRISMA flow-diagram shows the process of study selection and reasons for inclusion/exclusion (Figure 1). 


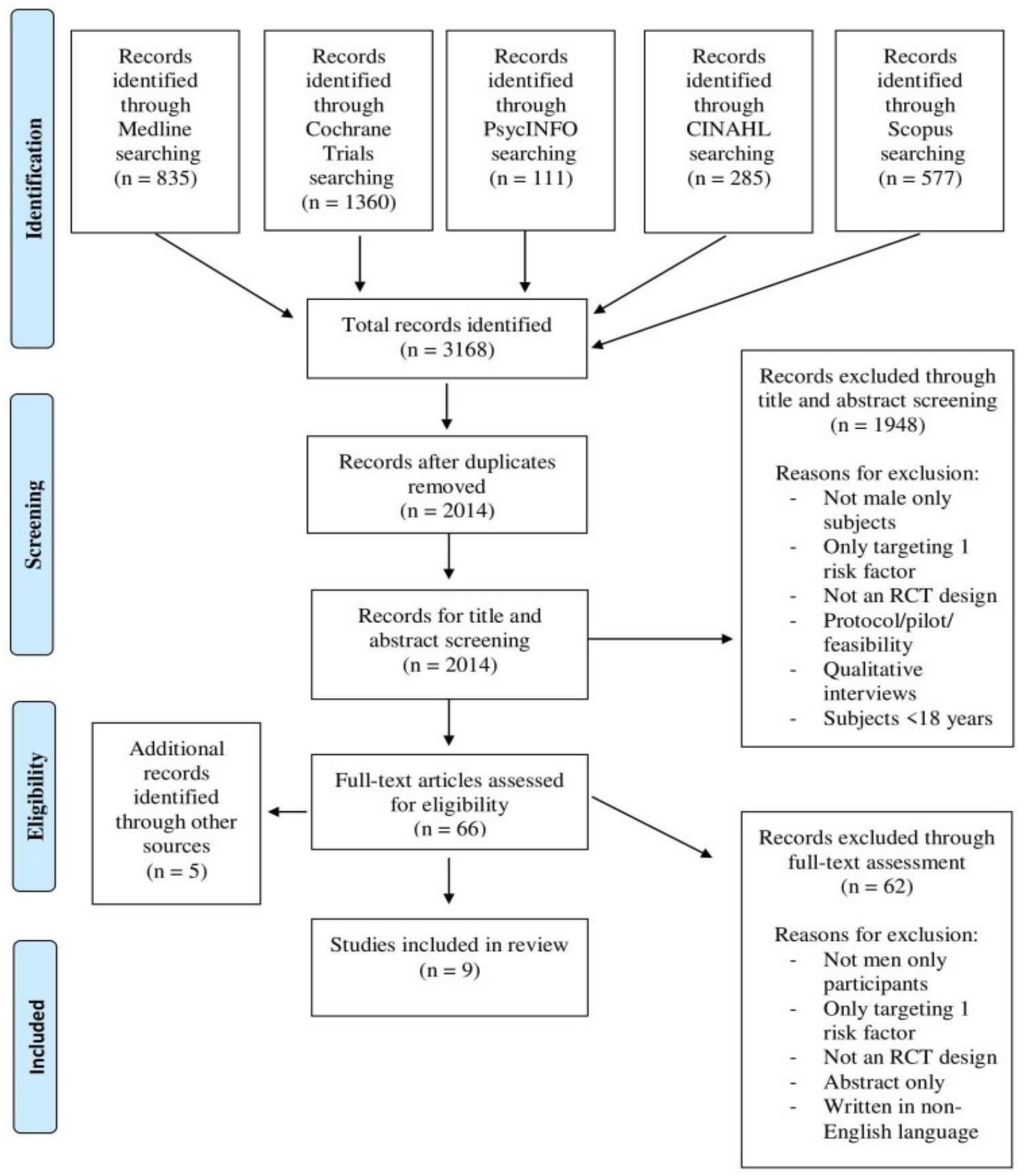

Figure 1. PRSIMA flow diagram 


\section{Study characteristics}

Of the nine trials reviewed (Table 2), five were conducted in Australia (Duncan et al., 2012; George, 2013; Morgan et al., 2009; 2011; 2013), three in the USA (Crane et al., 2015; Patrick et al., 2011; Veverka et al., 2003) and one in Japan (Tanaka et al., 2010). Six trials targeted men who were overweight or obese (Crane et al., 2015; Morgan et al.,2009; 2011; 2013; Patrick et al., 2011; Tanaka et al., 2010), two middle aged (Duncan et al., 2014; George, 2013) and one in the military (Veverka et al., 2003). One trial was a PhD thesis (George, 2013).

The main CVD-related outcomes assessed were weight (Crane et al., 2015; George, 2013; Morgan et al., 2009; 2011; 2013; Patrick et al., 2011; Tanaka et al., 2010; Veverka et al., 2003), physical activity (Crane et al., 2015; Duncan et al., 2014; George, 2013; Morgan et al., 2009; 2011; 2013; Patrick et al., 2011; Tanaka et al., 2010), diet/nutrition (Crane et al., 2015; Duncan et al., 2014; Morgan et al., 2009; 2011; 2013; Patrick et al., 2011; Tanaka et al., 2010) and related biomarkers/clinical measures: blood pressure, cholesterol, glucose, $\mathrm{HbA1c}$, resting heart rate and cardiorespiratory fitness $\left(\mathrm{VO}_{2} \mathrm{Max}\right)$ (George, 2013; Morgan et al., 2009; 2011; 2013; Tanaka et al., 2010; Veverka et al., 2003). Outcomes were reported as mean change scores in five studies (George, 2013; Morgan et al., 2009; 2011; 2013; Tanaka et al., 2010), pre-post means and SD or Cl within each group in three studies (Crane et al., 2015; Patrick et al., 2011; Veverka et al., 2003) and as exponentiated coefficients in one study (Duncan et al., 2012).

Of the nine trials, three incorporated an initial face-to-face session prior to engaging in the online component of the intervention to provide behavioral recommendations (Crane et al., 2015; Morgan et al., 2009; 2011). Most trials provided tailored feedback on behavioral habits (Crane et al., 2015; Duncan et al., 2014; Morgan et al., 2009, 2011, 2013; Patrick et al., 2011; Tanaka et al., 2010). To increase the success of the intervention in achieving behavior change, educating participants on goal setting (Crane et al., 2015; Duncan et al., 2014; George et al., 2013; Morgan et al., 2009; 2011; 2013; Patrick et al., 2011; Tanaka et al., 2010), self-monitoring/tracking progress (Crane et al., 2015; Duncan et al., 2014; George et al., 2013; 
Table 2. Characteristics of included studies

\begin{tabular}{|c|c|c|c|c|}
\hline $\begin{array}{l}\text { Author, } \\
\text { year, } \\
\text { country }\end{array}$ & $\begin{array}{l}\text { Study } \\
\text { design, } \\
\text { participants, } \\
\text { sample size, } \\
\text { age }\end{array}$ & Intervention & $\begin{array}{l}\text { CVD-related } \\
\text { outcomes }\end{array}$ & $\begin{array}{l}\text { Data collection time } \\
\text { points }\end{array}$ \\
\hline $\begin{array}{l}\text { Crane et al., } \\
2015 \\
\text { USA }\end{array}$ & $\begin{array}{l}\text { RCT, } \\
\text { overweight } \\
\text { or obese } \\
\text { men, } \\
\mathrm{n}=107, \\
\text { mean age = } \\
44.2 \text { years }\end{array}$ & $\begin{array}{l}\text { Theory use: yes, social cognitive theory and self-determination } \\
\text { theory } \\
\text { Duration: } 6 \text { months } \\
\text { Intended dose: weekly } \\
\text { IG: two 1-hour face-to-face group sessions for recommendations on } \\
\text { diet, physical activity, self-monitoring behaviors; future contact } \\
\text { delivered on-line; self-evaluation of behaviors for personalised } \\
\text { recommendations; automated tailored feedback; SMART goals set } \\
\text { each week; modest calorie deficit, minimum of } 6 \text { separate } 100- \\
\text { calorie changes; weekly online lesson on dietary strategies; } \\
\text { progressive exercise plan with target of } 225 \text { minutes' exercise per } \\
\text { week } \\
\text { CG: waitlist; no recommendations }\end{array}$ & $\begin{array}{l}\text { Body weight, BMI, } \\
\text { waist } \\
\text { circumference, diet, } \\
\text { physical activity }\end{array}$ & Baseline, 3, 6 months \\
\hline $\begin{array}{l}\text { Duncan et al., } \\
2014 \\
\text { Australia }\end{array}$ & $\begin{array}{l}\text { Two-armed } \\
\text { RCT, middle- } \\
\text { aged men, } \\
\mathrm{n}=301\end{array}$ & $\begin{array}{l}\text { Theory use: yes, social cognitive theory and self-regulation theory } \\
\text { Duration: } 9 \text { months }\end{array}$ & $\begin{array}{l}\text { Physical activity, } \\
\text { dietary behaviors }\end{array}$ & Baseline, 3, 9 months \\
\hline
\end{tabular}




\begin{tabular}{|c|c|c|c|c|}
\hline & $\begin{array}{l}\text { mean age }= \\
44 \text { years }\end{array}$ & $\begin{array}{l}\text { Intended dose: not stated } \\
\text { IG: website and mobile phone platform; materials on physical } \\
\text { activity, dietary behaviors, self-monitoring of behaviors; goal } \\
\text { setting; progress tracking; automated feedback on progress; social } \\
\text { support; 'Man-Up' challenges ( } 6 \text { on physical activity, } 1 \text { on healthy } \\
\text { eating) } \\
\text { CG: print-based materials covering the same topics provided to IG; } \\
\text { self-monitoring of behaviors }\end{array}$ & & \\
\hline $\begin{array}{l}\text { George et al., } \\
2013 \\
\text { Australia }\end{array}$ & $\begin{array}{l}\text { Two-armed } \\
\text { RCT, middle- } \\
\text { aged men } \\
\text { employed at } \\
\text { a university, } \\
\mathrm{n}=56, \\
\text { mean age = } \\
48.2 \text { years }\end{array}$ & $\begin{array}{l}\text { Theory use: no, social ecological approach to health promotion } \\
\text { Duration: } 3 \text { months } \\
\text { Intended dose: not stated } \\
\text { IG: adapted from initial Man-Up study (Duncan et al. 2012); 'Man- } \\
\text { Up challenges'; goal setting, progress tracking; 'Information centre' } \\
\text { for health advice; social forum; email reminders ( } 3,6,9 \text { weeks) to } \\
\text { access intervention; printed materials on physical activity, } \\
\text { sedentary time, health } \\
\text { CG: print-based comparison group, included materials used in } \\
\text { online intervention }\end{array}$ & $\begin{array}{l}\text { Physical activity, } \\
\text { sedentary time, } \\
\text { body weight, BMI, } \\
\text { waist } \\
\text { circumference, } \\
\text { blood pressure }\end{array}$ & Baseline, 3,6 months \\
\hline $\begin{array}{l}\text { Morgan et al., } \\
2009 \\
\text { Australia }\end{array}$ & $\begin{array}{l}\text { RCT, } \\
\text { overweight } \\
\text { or obese } \\
\text { men, } \\
n=65\end{array}$ & $\begin{array}{l}\text { Theory use: yes, social cognitive theory } \\
\text { Duration: } 3 \text { months } \\
\text { Intended dose: } 7 \text { individualised feedback sheets }\end{array}$ & $\begin{array}{l}\text { Body weight, BMI, } \\
\text { waist } \\
\text { circumference, } \\
\text { blood pressure, } \\
\text { physical activity, } \\
\text { dietary behaviors, }\end{array}$ & Baseline, 3, 6 months \\
\hline
\end{tabular}




\begin{tabular}{|c|c|c|c|c|}
\hline & $\begin{array}{l}\text { mean age }= \\
35.9 \text { years }\end{array}$ & $\begin{array}{l}\text { IG: initial face-to-face session covering diet, physical activity, } \\
\text { behavior change strategies (self-monitoring, goal setting, social } \\
\text { support); online website (CalorieKing) with information, tools to } \\
\text { improve diet, physical activity; progress tracking; weekly diaries for } \\
\text { first } 4 \text { weeks, } 2 \text { weeks in second month, } 1 \text { week in third month for } \\
\text { individualised feedback, encouragement, reinforcement; 'notice } \\
\text { board' for questions to research team } \\
\text { CG: initial information session, print-based resources relating to } \\
\text { online intervention }\end{array}$ & & \\
\hline $\begin{array}{l}\text { Morgan } \\
\text { et al., } 2011 \\
\text { Australia }\end{array}$ & $\begin{array}{l}\text { Two-armed } \\
\text { RCT, } \\
\text { overweight } \\
\text { or obese } \\
\text { men, } \\
\mathrm{n}=110, \\
\text { mean age = } \\
44.1 \text { years }\end{array}$ & $\begin{array}{l}\text { Theory use: yes, social cognitive theory } \\
\text { Duration: } 3 \text { months } \\
\text { Intended dose: } 7 \text { individualised feedback sheets } \\
\text { IG: modelled on SHED-IT program (Morgan et al. 2009): initial face- } \\
\text { to-face education session on energy balance, challenges of shift } \\
\text { work related to diet, exercise, weight loss tips for men, behavior } \\
\text { change strategies; online website (CalorieKing); submit weight } \\
\text { weekly; daily eating and exercise diary each week for first month, } \\
\text { twice in second month, once in third month for individualised } \\
\text { feedback, personalised weight loss strategies; self-monitoring, skills } \\
\text { for better weight loss behaviors, goal setting; weight loss handbook } \\
\text { and pedometer } \\
\text { CG: waitlist }\end{array}$ & $\begin{array}{l}\text { Body weight, waist } \\
\text { circumference, } \\
\text { BMI, blood } \\
\text { pressure, resting } \\
\text { heart rate, physical } \\
\text { activity, dietary } \\
\text { habits }\end{array}$ & Baseline, 14-weeks \\
\hline $\begin{array}{l}\text { Morgan } \\
\text { et al., } 2013 \\
\text { Australia }\end{array}$ & $\begin{array}{l}\text { Three-armed } \\
\text { RCT, } \\
\text { overweight } \\
\text { or obese } \\
\text { men, }\end{array}$ & $\begin{array}{l}\text { Theory use: yes, social cognitive theory } \\
\text { Duration: } 3 \text { months } \\
\text { Intended dose: } 7 \text { individualised feedback sheets }\end{array}$ & $\begin{array}{l}\text { Body weight, waist } \\
\text { circumference, } \\
\text { BMI, body fat } \\
\text { percentage, blood } \\
\text { pressure, resting }\end{array}$ & Baseline, 3, 6 months \\
\hline
\end{tabular}




\begin{tabular}{|c|c|c|c|c|}
\hline & $\begin{array}{l}\mathrm{n}=159 \\
\text { mean age = } \\
47.5 \text { years }\end{array}$ & $\begin{array}{l}\text { IG (print-based resources): community trial of SHED-IT program; } \\
\text { weight loss resource package targeted at men (DVD, handbook, } \\
\text { pedometer); physical activity, dietary behavior change, improve key } \\
\text { dietary behaviors using "masculinized" tips and strategies, weight } \\
\text { loss tips, three SMART goals per month } \\
\text { IG (online + print-based resources): same materials as print-based } \\
\text { intervention group; online website (CalorieKing), input food and } \\
\text { exercise diary } 4 \text { days per week; goal setting, progress tracking, } \\
\text { individualised feedback via email ( } 7 \text { in total) based on diary entries; } \\
\text { strategies for improving diet and exercise behaviors } \\
\text { CG: waitlist }\end{array}$ & $\begin{array}{l}\text { heart rate, physical } \\
\text { activity, sedentary } \\
\text { time, dietary habits }\end{array}$ & \\
\hline $\begin{array}{l}\text { Patrick et al., } \\
2011 \\
\text { USA }\end{array}$ & $\begin{array}{l}\mathrm{RCT}, \\
\text { overweight } \\
\text { or obese } \\
\text { men, } \\
\mathrm{n}=441, \\
\text { mean age = } \\
43.9 \text { years }\end{array}$ & $\begin{array}{l}\text { Theory use: yes, social cognitive theory } \\
\text { Duration: } 12 \text { months } \\
\text { Intended dose: weekly } \\
\text { IG: weight loss through diet, physical activity; goal setting, } \\
\text { behavioral skills, social support; improving fruit and vegetable } \\
\text { intake, reducing saturated fat, increasing whole grain products; } \\
\text { 10,000 steps } 5 \text { times per week, strength training } 2 \text { times per week; } \\
\text { computerised assessment for personalised recommendations; } \\
\text { weekly web-based activities; pedometers; frequent reassessment } \\
\text { for further personalised advice } \\
\text { CG: waitlist, alternate website for general health information }\end{array}$ & $\begin{array}{l}\text { Body weight, BMI, } \\
\text { waist } \\
\text { circumference, diet, } \\
\text { physical activity }\end{array}$ & Baseline, 6, 12 months \\
\hline
\end{tabular}




\begin{tabular}{|c|c|c|c|c|}
\hline $\begin{array}{l}\text { Tanaka et al., } \\
2013 \\
\text { Japan }\end{array}$ & $\begin{array}{l}\mathrm{RCT}, \\
\text { overweight } \\
\text { men, } \\
\mathrm{n}=51, \\
\text { mean age = } \\
46 \text { years }\end{array}$ & $\begin{array}{l}\text { Theory use: not stated } \\
\text { Duration: } 1 \text { month } \\
\text { Intended dose: not stated } \\
\text { IG: educational booklet on behavioral weight control, diet, physical } \\
\text { activity, self-monitoring with examples; questionnaire for food, } \\
\text { physical activity habits, input to "KT" computer system for } \\
\text { personalised advice, behavioral techniques, encouragement, skills } \\
\text { for change, goal setting, self-monitoring advice; second } \\
\text { questionnaire after one month for further advice; understanding } \\
\text { barriers, reinforcement, social support } \\
\text { CG: received same booklet as IG }\end{array}$ & $\begin{array}{l}\text { Body weight, BMI, } \\
\text { blood pressure, } \\
\text { blood cholesterol } \\
\text { (Total, LDL, HDL, } \\
\text { triglycerides), } \\
\text { glucose, HbA1c, } \\
\text { physical activity, } \\
\text { dietary behaviors }\end{array}$ & $\begin{array}{l}\text { Baseline, 1, 3, } 7 \\
\text { months }\end{array}$ \\
\hline $\begin{array}{l}\text { Veverka et } \\
\text { al., } 2003 \\
\text { USA }\end{array}$ & $\begin{array}{l}\mathrm{RCT}, \\
\text { men on } \\
\text { active } \\
\text { military duty, } \\
\mathrm{n}=39 \\
\text { mean age = } \\
\text { not stated }\end{array}$ & $\begin{array}{l}\text { Theory use: yes, transtheoretical model of behavior change } \\
\text { Duration: } 6 \text { months } \\
\text { Intended dose: minimum once per month } \\
\text { IG: online survey for participants to be staged, received information } \\
\text { on both exercise and diet } \\
\text { CG: control, no intervention or materials }\end{array}$ & $\begin{array}{l}\text { Cardiorespiratory } \\
\text { fitness }\left(\mathrm{VO}_{2} \text { max), }\right. \\
\text { body weight, body } \\
\text { fat percentage, } \\
\text { BMI, waist-hip- } \\
\text { ratio), blood } \\
\text { cholesterol (Total, } \\
\text { LDL, HDL, } \\
\text { triglycerides), blood } \\
\text { pressure, resting } \\
\text { heart rate }\end{array}$ & Baseline, 6 months \\
\hline
\end{tabular}

IG: intervention group; CG: control group 
Morgan et al., 2009; 2011; 2013; Tanaka et al., 2010) and social interaction/support (Duncan et al., 2014; George et al., 2013; Morgan et al., 2009; Patrick et al., 2011; Tanaka et al., 2010) was included. In addition to the online component of the intervention, participants in four trials (George et al., 2013; Morgan et al., 2011; 2013; Tanaka et al., 2010) were provided with supplementary resources (i.e. handbooks, DVD, printed materials). One trial (Veverka et al., 2003) provided little to no information on the characteristics of their intervention.

Of the interventions reported in the nine trials, seven were underpinned by theory, two of which used two theories. Six interventions were underpinned by social cognitive theory (Crane et al., 2015; Duncan et al., 2014; Morgan et al., 2009; 2011; 2013; Patrick et al., 2011), - one in combination with self-determination theory (Crane et al., 2015) and one with selfregulation (Duncan et al., 2014) - and one by the transtheoretical model of behavior change (Veverka et al., 2003). One trial (George, 2013) took an ecological approach to health promotion rather than targeting a single theoretical framework, and one did not state whether the intervention was driven by theory (Tanaka et al., 2010).

The choice of comparison groups in the nine trials varied: four used a wait-list control group (Crane et al., 2015; Morgan et al., 2011; 2013; Patrick et al., 2011), four used a comparison group which received printed resources related to the online intervention, (Duncan et al., 2014; George, 2013; Morgan et al., 2009; Tanaka et al., 2010) and one used a 'true control' group, i.e. receiving no intervention or materials (Veverka et al., 2003). Mean participant age across the nine trials was $44.2 \pm 3.8$ years with one trial (Veverka et al., 2003) not reporting age. Study sample sizes ranged from 39-441 at the point of randomisation. Intervention durations ranged between one and 12-months with participant retention rates at the final assessment point ranging between $49.2 \%$ and $93 \%$ with an average of $79.3 \% \pm$ 13.6\%. Table 2 shows a summary of the study characteristics.

\section{Quality of studies}

Overall, risk of bias across the included trials was rated as low (Duncan et al., 2014; George, 2013; Morgan et al., 2009; 2011; 2013). Three trials had an unclear risk of bias (Patrick et al., 
2011; Tanaka et al., 2010; Veverka et al., 2003), and one a high risk of bias (Crane et al., 2015). Figure 2 shows a summary of the risk of bias assessment.

In most trials (Crane et al., 2015; Duncan et al., 2014; George, 2013; Morgan et al., $2009 ; 2011 ; 2013$; Patrick et al., 2011) the process of randomisation to intervention and control groups was described, thus presenting a low risk of bias (random sequence generation). Allocation concealment to groups was reported in six trials (Crane et al., 2015; Duncan et al., 2014; George, 2013; Morgan et al., 2009; 2011; 2013), but was unclear in three (Patrick et al., 2011; Tanaka et al., 2010; Veverka et al., 2003). The blinding of participants and personnel was unclear in four trials (Crane et al., 2015; Patrick et al., 2011; Tanaka et al., 2010; Veverka et al., 2003). The blinding of outcome assessment varied among trials: the outcome assessor was blinded throughout (low risk of bias) in four (Duncan et al., 2014; George, 2013; Morgan et al., 2009; 2011; 2013; Patrick et al., 2011), two lacked sufficient detail (unclear) (Tanaka et al., 2010; Veverka et al., 2003), and one (Crane et al., 2015) was rated as having a high risk of bias due to non-blinding of the outcome assessor. Risk of bias for both the incomplete outcome data and selective reporting domains was rated as low for all trials.

As identified by the meta-analyses, considerable heterogeneity was present for BMI, body weight and waist circumference (see below), therefore the findings for these should be interpreted with caution. The heterogeneity observed appears to be as a result of study sample sizes as measurement tools were the same for each outcome across trials. No concern about heterogeneity was observed for either systolic or diastolic blood pressure. 
Risk of bias domains

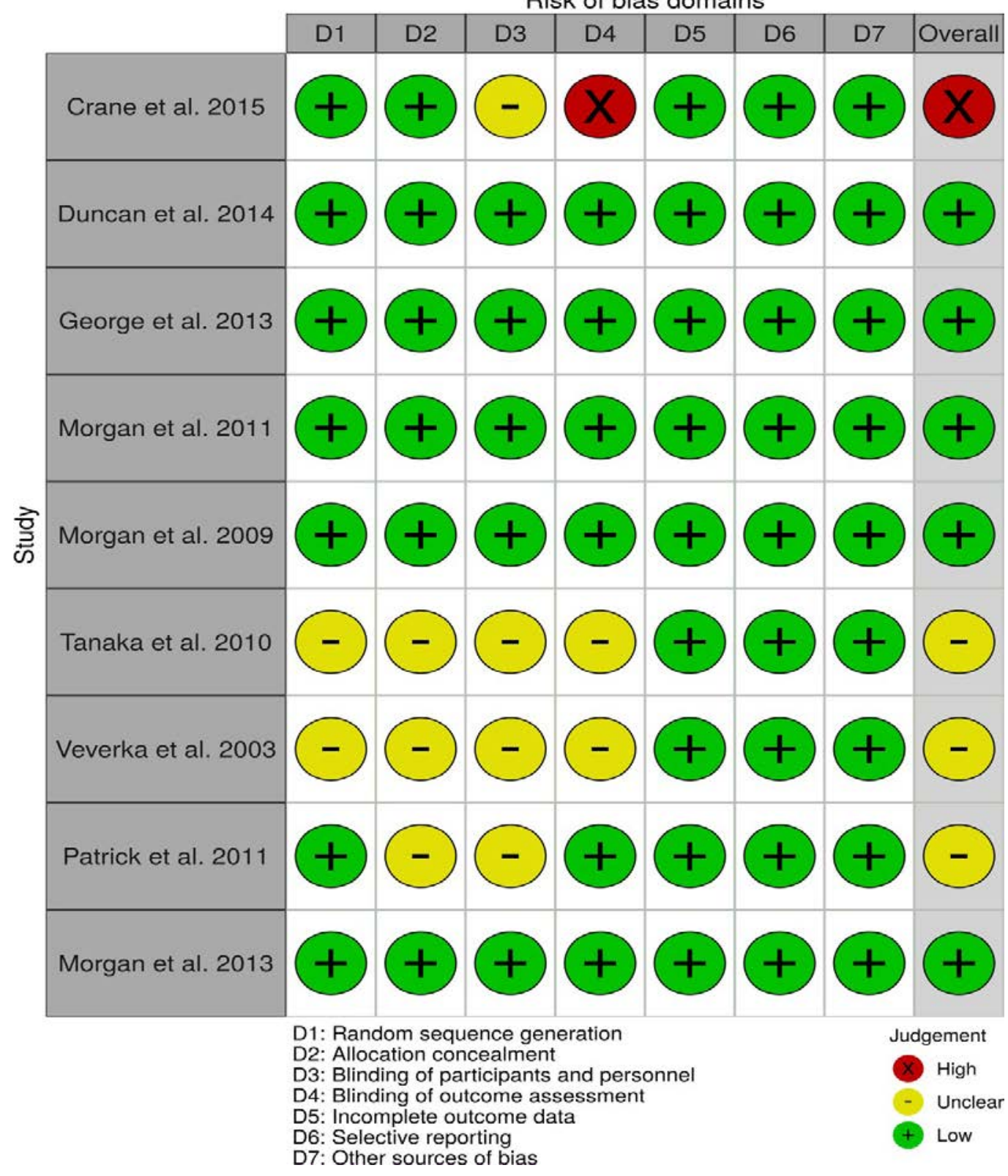

Figure 2. Summary of risk of bias assessment. Created using the robvis web application (McGuinness, 2019) 


\section{Meta-analysis}

Body mass index

BMI (Figure 3), measured at post-intervention in seven trials, was reduced by $0.64 \mathrm{~kg} / \mathrm{m}^{2}$ in the online intervention group compared to a control or resource-based comparison group, $Z=-2.75, p=0.01, l^{2}=76.39$. One trial removed from the analysis did not significantly change the study outcome.

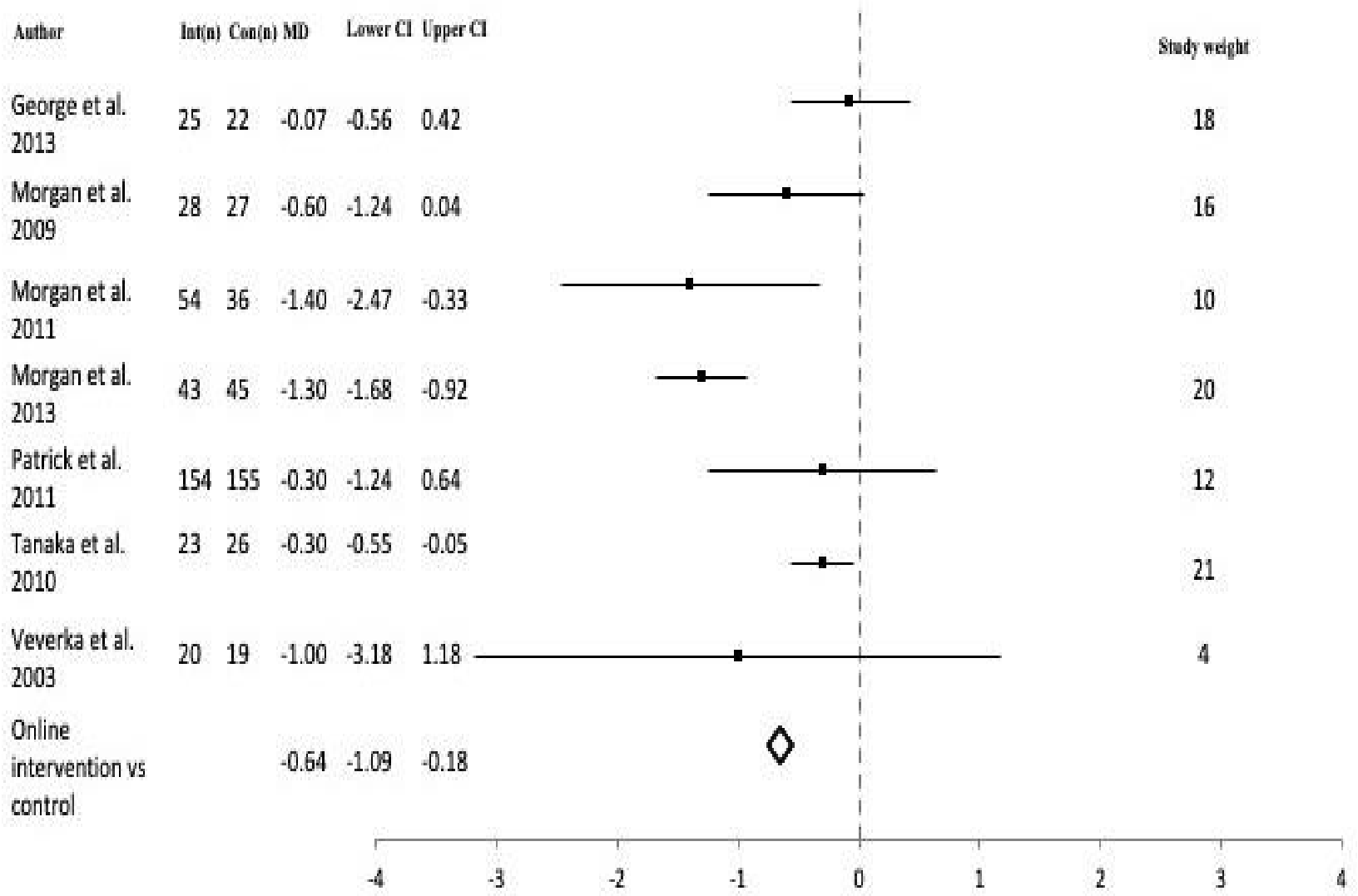

Figure 3. Forest plot - effect of online intervention vs control for BMI $\left(\mathrm{kg} / \mathrm{m}^{2}\right)$ 


\section{Body weight}

Body weight (Figure 4), measured at post-intervention in eight trials, was reduced by $2.27 \mathrm{~kg}$ in the online intervention group compared to a control or resource-based comparison group, $Z=-3.25, p<0.01, I^{2}=77.34$. One study removed from the analysis did not significantly change the study outcome.

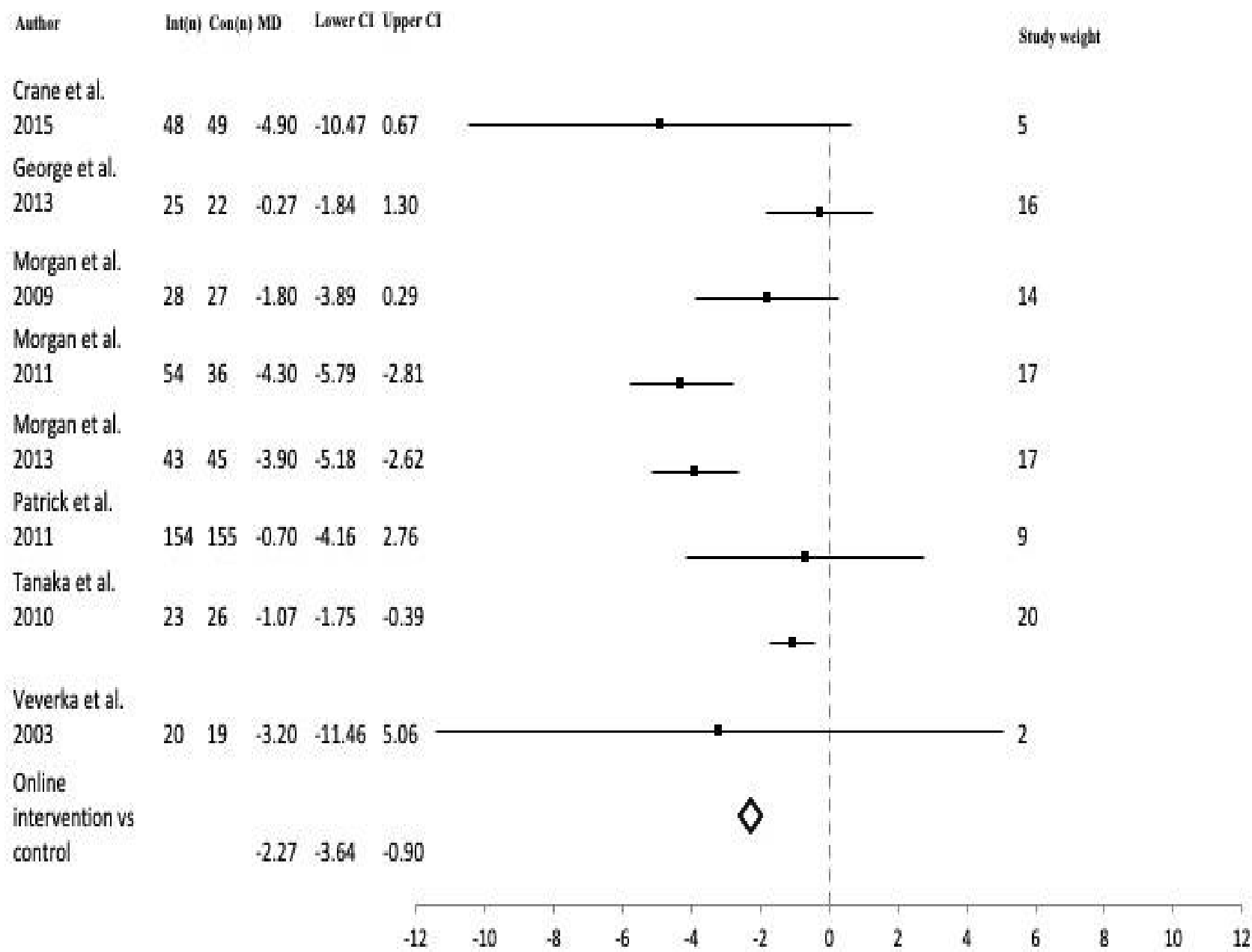

Figure 4. Forest plot - effect of online intervention vs control for body weight $(\mathrm{kg})$ 


\section{Waist circumference}

Waist circumference (Figure 5), measured at post-intervention in six trials, was reduced by $2.46 \mathrm{~cm}$ in the online intervention group compared to a control or resource-based comparison group, $Z=-2.30 p=0.02, l^{2}=84.64$. Removal of the studies by Morgan et al. $(2011,2013)$ resulted in p-values of 0.09 and 0.12 , respectively.

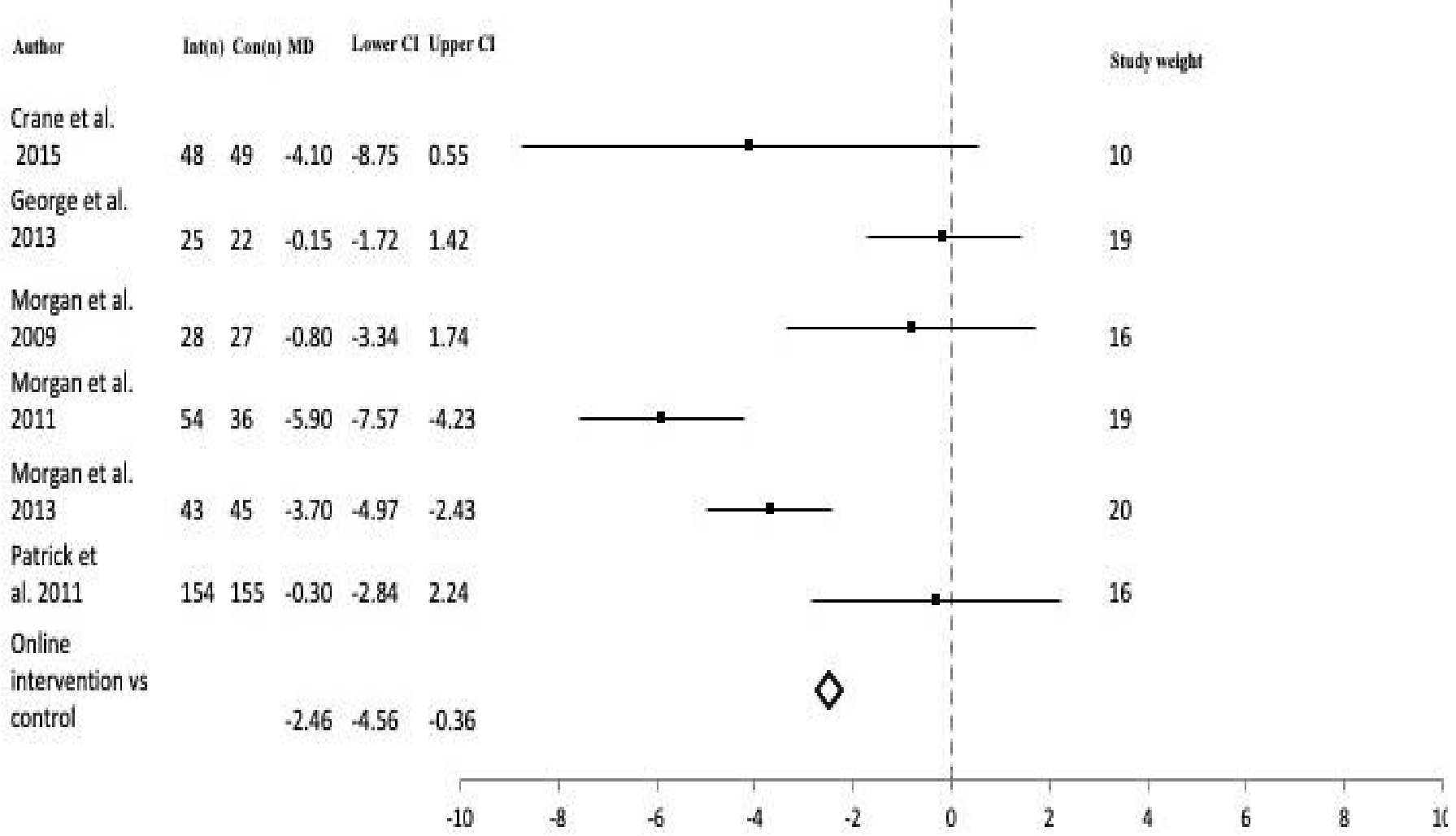

Figure 5. Forest plot - effect of online intervention vs control for waist circumference $(\mathrm{cm})$ 
Systolic blood pressure

Systolic blood pressure (Figure 6), measured at post-intervention in six trials, was reduced by $4.22 \mathrm{mmHg}$ in the online intervention group compared to a control or resource-based comparison group, $Z=-3.57, p<0.01, l^{2}=0$. One study removed from the analysis did not significantly change the study outcome.

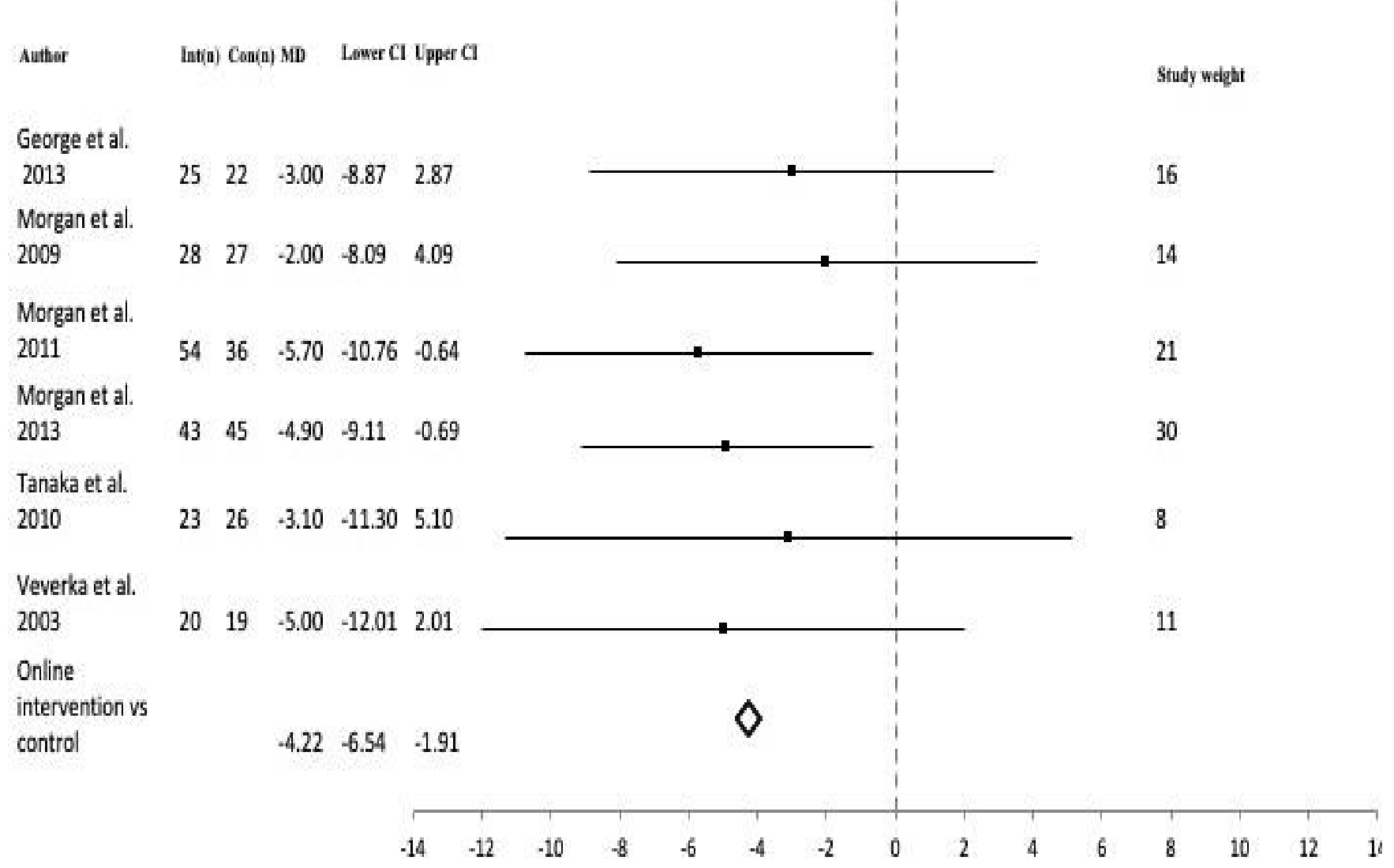

Figure 6. Forest plot - effect of online intervention vs control for systolic blood pressure $(\mathrm{mmHg})$ 


\section{Diastolic blood pressure}

Diastolic blood pressure (Figure 7), measured at post-intervention in six trials, was reduced by $2.87 \mathrm{mmHg}$ in the online intervention group compared to a control or resource-based comparison group, $Z=-3.56, p<0.01, I^{2}=0$. One study removed from the analysis did not significantly change the study outcome.

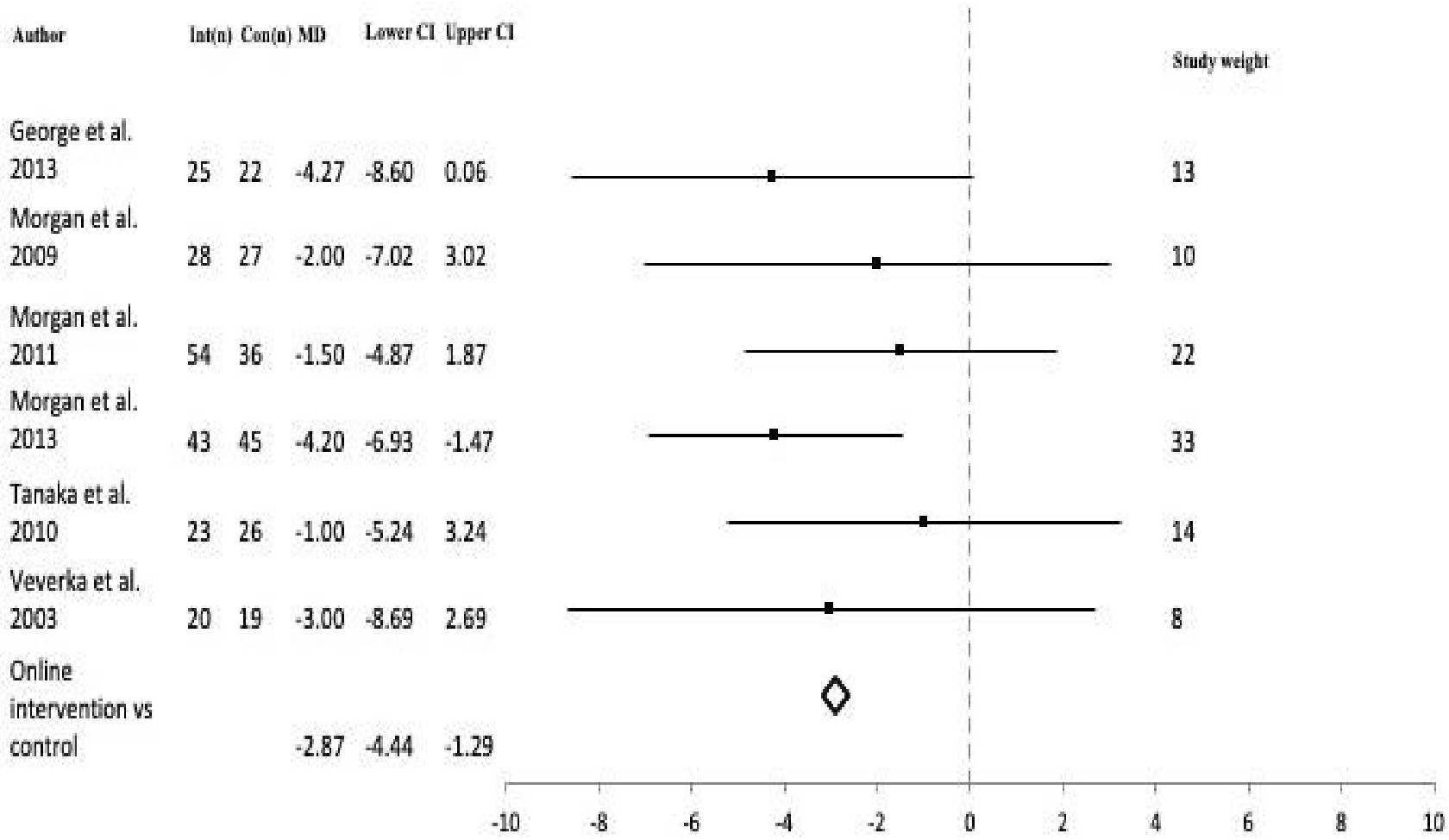

Figure 7. Forest plot - effect of online intervention vs control for diastolic blood pressure $(\mathrm{mmHg})$ 


\section{Narrative synthesis}

\section{Behavioral risk factors}

Physical activity and sedentary time

Physical activity was assessed in eight of the nine trials (Crane et al., 2015; Duncan et al., 2014; George, 2013; Morgan et al., 2009; 2011; 2013; Patrick et al., 2011; Tanaka et al., 2010). Six trials reported improvements in physical activity related outcomes in favour of those in the online intervention group, four of which were statistically significant. Two trials reported time spent sedentary: one measured sedentary time objectively, reporting no change in either group across time-points; the other trial measured sedentary time subjectively, reported nonsignificant improvements.

\section{Diet}

Diet was assessed by self-report in seven of the nine trials. Measurement tools varied across trials with the most common tools used being the food frequency questionnaire $(n=3)$. Dietary outcomes included: calorie intake (Crane et al., 2015; Morgan et al., 2009; 2013), consumption of bread (Duncan et al., 2014; Morgan et al., 2011), milk (Duncan et al., 2014; Morgan et al., 2011), beverages (Morgan et al., 2011) and alcohol (Morgan et al., 2013). Fruit and vegetable intake were assessed in two trials (Morgan et al., 2011; Patrick et al., 2011) with the percentage of energy from dietary fats and saturated fats assessed in one trial (Patrick et al., 2011). One trial (Tanaka et al., 2010) reported on the intake of processed and fat rich foods, as well as dietary behaviors exhibited by participants. Lastly, portion size was assessed in one trial (Morgan et al., 2013). Five trials reported improvements for dietary outcomes with significant differences between groups observed in one study only.

\section{Biomarkers/clinical measures}

Resting heart rate

Four trials assessed change in resting heart rate (Morgan et al., 2009; 2011; 2013; Veverka et al., 2003) with improvements observed across all studies post-intervention compared to baseline. Two reported significant improvements in resting heart rate from baseline in the 
online intervention group with one (Morgan et al., 2011) reporting a significant difference between the online intervention and control group (mean difference between groups $=7.9$ bpm).

Blood lipids, glucose and HbA1C

Two trials (Tanaka et al., 2010; Veverka et al., 2003) measured blood lipids and reported improvements in total, HDL and LDL cholesterol for the intervention and control groups and triglycerides in both intervention groups, with the control groups observing increases in triglycerides during the study period. One trial (Veverka et al., 2003) reported greater improvements in all cholesterol measurements in favour of the intervention group. However, the other trial (Tanaka et al., 2010) reported that improvements in total and HDL cholesterol were only greater than the control group at the 1-month assessment, with the control group observing greater reductions than the intervention group at the 3-month assessment point.

Only one trial measured blood glucose and HbA1c levels (Tanaka et al., 2010). Although both the intervention and control groups observed reductions in blood glucose, the online intervention group had greater reductions than the control. HbA1c was reduced at both time points for the intervention group whereas the control group only observed improvements during the 3-month assessment point. There were no significant differences observed between groups for either blood cholesterol, blood glucose or HbA1c.

\section{Cardiorespiratory fitness}

One trial assessed change in cardiorespiratory fitness through Vo2 Max testing (Veverka et al., 2003). Although Vo2 Max scores improved in both groups and more in the intervention group, there was no significant difference between the two groups.

\section{Discussion}

To our knowledge, this is the first systematic review and meta-analysis of RCTs of eHealth interventions for reducing CVD risk factors in men. The review found improvements in body weight, BMI, waist circumference and blood pressure among men receiving eHealth interventions compared to a control or printed resources comparison group. Less evident 
were improvements in physical activity and diet. Although many of these improvements were at a statistical level of significance their clinical significance is likely to be modest.

Our findings compare favourably with reviews of eHealth interventions for CVD prevention that are not for men only - for example, Widmer et al. (2015) found significant reductions in weight and BMI but not blood pressure or other CVD risk factors - as well as for overweight/obese adults (Hutcheson et al., 2015; Lau et al., 2020), physical activity and diet (Kroeze et al., 2006; Norman et al., 2007), or physical activity alone (Davies et al., 2012; Jahangiry et al., 2017).

Even though eHealth interventions are assuming increasing prominence and appeal, they often suffer from poor rates of engagement, uptake and adherence (Kelders et al., 2012). Regarding participant retention, five of the nine trials reported rates exceeding $80 \%$, three exceeding $90 \%$ at follow-up, indicating acceptability of eHealth interventions among men. Also, a wait-list control group is a potential mechanism to increase the acceptability of being randomised to a control group and improve retention rates (Darker et al., 2009). This is supported by our review, with the highest attrition rates observed in three of the four trials that used an 'active control': a comparison group provided with printed resources (Duncan et al., 2014; George, 2013; Morgan et al., 2013).

The review found poor adherence rates with the online intervention, with only one trial (Crane et al., 2015) reporting positive rates, with a mean of $90 \%$ during the first 12 -weeks, declining to $77 \%$ from 12 to 22 -weeks.

Trials utilising an 'active control' found greater rates of attrition and fewer mean differences between groups for CVD-related outcomes than those utilising a 'true control'. Using an 'active control' has the potential to negatively impact the perceived benefits brought about by the online intervention by minimizing the differences between groups compared to using a 'true control' (Möller, 2011). As indicated in our review, it would be advisable to approach the mean differences between groups in these trials with caution as the true effect of the online intervention may not be as evident. 
Seven trials reported the use of a theory-based intervention to enhance behavior change. The application of behavior change theories is strongly advocated by Medical Research Council guidance on developing and evaluating complex interventions (Craig et al., 2008). Previous reviews of eHealth behavior change interventions found that those driven by a theoretical basis were more effective than those that were not (Muellmann et al., 2016; Webb et al., 2010). It is difficult to ascertain the influence of behavior change theories on outcomes in this review, and we can merely speculate, though the trial (George, 2013) which utilised an ecological approach to health promotion rather than a specific behavior change theory produced the least difference in change between groups for all weight-related outcomes.

Finally, it is important to note that earlier, non-eHealth, reviews of counselling or education interventions (usually face-to-face or group) for primary prevention of CVD have found, at best, only modest declines in risk factors (Ebrahim et al., 2011; Alageel et al., 2017), and we reiterate the plea made by the authors of these reviews for better reporting of the rationale, content and delivery of interventions in order to understand their effectiveness. For example, none of the trials in our review examined the association between intervention characteristics and outcomes, an issue that should be explored in future trials.

\section{Limitations}

There are a number of limitations to this review. First, due to a lack of translation resources it is possible that relevant studies in a language other than English may have been missed, and more comprehensive reviews addressing this gap are warranted. Second, although improvements were observed for body weight, BMI and waist circumference in statistically significant terms, there was considerable heterogeneity between studies. Third, and related to the second, is the challenge in determining whether such improvements are clinically significant. Fourth, three of the trials were from the same group and overlapped, thus potentially skewing the findings. Fifth, three trials were assessed as having an unclear risk of bias, which points to the need for studies to report clearly and unambiguously issues such as group allocation and blinding. Sixth, we were unable to determine the association between intervention characteristics and outcomes, an issue warranting further exploration. 


\section{Conclusion}

The findings of this review highlight the potential effectiveness of eHealth interventions for reducing a range CVD risk factors in men. However, male participant adherence is an issue that warrants being addressed in future studies. Importantly, researchers should also ensure that eHealth interventions are developed with a theoretical underpinning to help understand their effectiveness in this population.

\section{Acknowledgements}

This work was supported by the Department for the Economy.

\section{References}

Alageel, S., Gulliford, M.C., McDermott, L., Wright, A.J., 2017. Multiple health behaviour change interventions for primary prevention of cardiovasculasr disease in primary care: systematic review and meta-analysis. BMJ Open 7, e015375.

Borenstein, M., Hedges, L.V., Higgins, J.P.T., Rothstein, H.R., 2009. Introduction to Meta-Analysis. John Wiley \& Sons, Chichester.

Bottorff, J.L., Seaton, C.L., Johnson, S.T., Caperchione, C.M., Oliffe, J.L., More, K., Jaffer-Hirji, H., Tillotson, S.M., 2015. An updated review of interventions that include promotion of physical activity for adult men. Sports Medicine 45, 775-800.

Craig, P., Dieppe, P., Macintyre, S., Michie, S., Nazareth, I., Petticrew, M., 2008. Developing and evaluating complex interventions: the new Medical Research Council guidance. BMJ 337, a1655.

Crane, M.M., Lutes, L.D., Ward, D.S., Bowling, J.M., Tate, D.F., 2015. A randomized trial testing the efficacy of a novel approach to weight loss among men with overweight and obesity. Obesity 23, 2398-405.

Darker, C.D., French, D., Eves, F.F., Sniehotta, F.F., 2009. An intervention to promote walking amongst the general population based on an 'extended' Theory of Planned Behavior: a waiting list randomized controlled trial. Psychology \& Health 25, 71-88. 
Davies, C.A., Spence, J.C., Vandelanotte, C., Caperchione, C.M., Mummery, W., 2012. Meta-analysis of internet-delivered interventions to increase physical activity levels. International Journal of Behavioral Nutrition and Physical Activity 9, 52.

De Vries, H., Brug, J., 1999. Computer-tailored interventions motivating people to adopt health promoting behaviours: introduction to a new apparoach. Patient Education and Counseling 36, 99-105.

Drew, R.J., Morgan, P.J., Pollock, E.R., Young, M.D., 2020. Impact of male-only lifestyle interventions on men's mental health: a systematic review and meta-analysis. Obesity Reviews 21, e13014.

Duncan, M., Vandelanotte, C., Kolt, G.S., Rosenkranz, R.R., Caperchione, C.M., George, E.S., Ding, H., Hooker, C., Karunanithi, M., et al., 2014. Effectiveness of a web- and mobile phone-based intervention to promote physical activity and healthy eating in middle-aged males: randomized controlled trial of the ManUp study. Journal of Medical Internet Research 16, e136.

Duncan, M.J., Vandelanotte, C., Rosenkranz, R.R., Caperchione, C.M., Ding, H., Ellison, M., George, E.S., Hooker, C., Karunanithi, M., et al., 2012. Effectiveness of a website and mobile phone based physical activity and nutrition intervention for middle-aged males: trial protocol and baseline findings of the ManUp study. BMC Public Health $12,656$.

Ebrahim, S., Taylor, F., Ward, K., Beswick, A., Burke, M., Davey Smith, G., 2011. Multiple risk factor interventions for primary prevention of coronary heart disease (Review). Cochrane Database of Systematic Reviews, 1, CD001561.

Gavarkovs, A.G., Burke, S.M., Petrella, R.J., 2016. Engaging men in chronic disease prevention and management programs: a scoping review. American Journal of Men's Health 10, N145-N54.

George, E.S., 2013. Increasing physical activity and reducing sedentary time in middle-aged males. PhD dissertation, University of Western Sydney.

Higgins, J.P.T., Altman, D.G., Gotzsche, P.C., Juni, P., Moher, D., Oxman, A.D., Savovic, J., 
Schulz, K.F., Weeks, L., et al., 2011. The Cochrane Collaboration's tool for assessing risk of bias in randomised trials. BMJ 343, d5928-d28.

Higgins, J.P.T., Thomas, J., Chandler, J., Cumpston, M., Li, T., Page, M.J., Welch, V.A., 2019. Cochrane Handbook for Systematic Reviews of Interventions, 2nd ed. John Wiley \& Sons, Chichester.

Hutchesson, M.J., Rollo, M.E., Krukowski, R., Ells, L., Harvey, J., Morgan, P.J., Callister. R., Plotnikoff, R., Collins, C.E, 2015. eHealth interventions for the prevention and treatment of overweight and obesity in adults: a systematic review with metaanalysis. Obesity Reviews 16, 376-92.

Jahangiry, L., Farhangi, M.A., Shab-Bidar, S., Rezaei, F., Pashaei, T., 2017. Web-based physical activity interventions: a systematic review and meta-analysis of randomized controlled trials. Public Health 152, 36-46.

Kelders, S.M., Kok, R.N., Ossebaard, H.C., Van Gemert-Pijnen, J.E., 2012. Persuasive system design does matter: a systematic review of adherence to web-based interventions. Journal of Medical Internet Research 14, e152.

Kroeze, W., Werkman, A., Brug, J., 2006. A systematic review of randomized trials on the effectiveness of computer-tailored education on physical activity and dietary behaviors. Annals of Behavioral Medicine 31, 205-23.

Lau, Y., Chee, D.G.H., Chow, X.P., Cheng, L.J., Wong, S.N., 2020. Personalised eHealth interventions in adults with overweight and obesity: a systematic review and metaanalysis of randomised controlled trials. Preventive Medicine 132,106001.

Malik, S.H., Blake, H., Suggs, L.S., 2014. A systematic review of workplace health promotion interventions for increasing physical activity. British Journal of Health Psychology 19, 149-80.

Martin, A., Fitzsimons, C., Jepson, R., Saunders, D.H., Van Der Ploeg, H.P., Teixeira, P.J., Gray, C.M., Mutrie, N., 2015. Interventions with potential to reduce sedentary time in adults: systematic review and meta-analysis. British Journal of Sports Medicine 49, 1056-63. 
McGuinness, L.A., 2019. robvis: an R package and web application for visualising risk-of-bias assessments. https://github.com/mcguinlu/robvis

Moher, D., Liberati, A., Tetzlaff, J., Altman, D.G., PRISMA Group, 2009. Preferred reporting items for systematic reviews and meta-analyses: the PRISMA statement. Annals of Internal Medicine 151, 264-69.

Moinuddin, A., Goel, A., 2016. Alcohol consumption and gender: a critical review. Journal of Psychology \& Psychotherapy 6, 1-4.

Möller, H.-J., 2011. Effectiveness studies: advantages and disadvantages. Dialogues in Clinical Neuroscience 13, 199-207.

Morgan, P.J., Callister, R., Collins, C.E., Plotnikoff, R.C., Young, M.D., Berry, N., McElduff, P., Burrows, T., Aguiar, E., et al., 2013. The SHED-IT community trial: a randomized controlled trial of internet- and paper-based weight loss programs tailored for overweight and obese men. Annals of Behavioral Medicine 45, 139-52.

Morgan, P.J., Collins, C.E., Plotnikoff, R.C., Cook, A.T., Berthon, B., Mitchell, S., Callister, R., 2011. Efficacy of a workplace-based weight loss program for overweight male shift workers: the Workplace POWER (Preventing Obesity Without Eating like a Rabbit) randomized controlled trial. Preventive Medicine 52, 317-25.

Morgan, P.J., Lubans, D.R., Collins, C.E., Warren, J.M., Callister, R., 2009. The SHED-IT randomized controlled trial: evaluation of an Internet-based weight-loss program for men. Obesity 17, 2025-32.

Muellmann, S., Forberger, S., Möllers, T., Zeeb, H., Pischke, C.R., 2016. Effectiveness of eHealth interventions for the promotion of physical activity in older adults: a systematic review protocol. Systematic Reviews 5, 47.

Norman, G.J., Zabinski, M.F., Adams, M.A., Rosenberg, D.E., Yaroch, A.L., Atienza, A.A., 2007. A review of eHealth interventions for physical activity and dietary behavior change. American Journal of Preventive Medicine 33, 336-45.

Pagoto, S.L., Schneider, K.L., Oleski, J.L., Luciani, J.M., Bodenlos, J.S., Whited, M.C., 
2012. Male inclusion in randomized controlled trials of lifestyle weight loss interventions. Obesity 20, 1234-9.

Patrick, K., Calfas, K.J., Norman, G.J., Rosenberg, D., Zabinski, M.F., Sallis, J.F., Rock, C.L., Dillon, L.W., 2011. Outcomes of a 12-month web-based intervention for overweight and obese men. Annals of Behavioral Medicine 42, 391-401.

Tanaka, M., Adachi, Y., Adachi, K., Sato, C., 2010. Effects of a non-face-to-face behavioral weight-control program among Japanese overweight males: a randomized controlled trial. International Journal of Behavioral Medicine 17, 17-24.

Turco, J.V., Inal-Veith, A., Fuster, V., 2018. Cardiovascular health promotion: an issue that can no longer wait. Journal of the American College of Cardiology 72, 908-13.

van Gemert-Pijnen, L., Kelders, S.M., Kip, H., Sanderman, R., 2018. eHealth Research, Theory and Development: A Multidisciplinary Approach. Routledge, London.

Vari, R., Scazzocchio, B., D'Amore, A., Giovannini, C., Gessani, S., Masella, R., 2016. Gender-related differences in lifestyle may affect health status. Annali dell'Istituto Superiore di Sanità 52, 158-66.

Veverka, D.V., Anderson, J., Auld, G.W., Coulter, G.R., Kennedy, C., Chapman, P.L., 2003. Use of the Stages of Change Model in improving nutrition and exercise habits in enlisted Air Force men. Military Medicine 168, 373-79.

Virani, S.S., Alonso, A., Benjamin, E.J., Bittencourt, M.S., Callaway, C.W., Carson, A.P., Chamberlain, A.M., Chang, A.R., Cheng, S., et al., 2020. Heart disease and stroke statistics - 2020 update: a report from the American Heart Association. Circulation 141, e139-e596.

Webb, T.L., Joseph, J., Yardley, L., Michie, S., 2010. Using the Internet to promote health behavior change: a systematic review and meta-analysis of the impact of theoretical basis, use of behavior change techniques, and mode of delivery on efficacy. Journal of Medical Internet Research 12, e4.

White, A., McKee, M., Richardson, N., De Visser, R., Madsen, S.A., De Sousa, B.C., 
Hogston, R., Zatoński, W., Makara, P., 2011. Europe's men need their own health strategy. BMJ 343, d7397.

Widmer, R.J., Collins, N.M., Collins, C.S., West, C.P., Lerman, L.O., Lerman, A., 2015. Digital health interventions for the prevention of cardiovascular disease: a systematic review and meta-analysis. Mayo Clinic Proceedings 90, 469-480.

World Health Organization, 2017. Cardiovascular diseases (CVDs). https://www.who.int/en/news-room/fact-sheets/detail/cardiovascular-diseases-(cvds)

Young, M.D., Morgan, P.J., Plotnikoff, R.C., Callister, R., Collins, C.E., 2012. Effectiveness of male-only weight loss and weight loss maintenance interventions: a systematic review with meta-analysis. Obesity Reviews 13, 393-408.

Yousaf, O., Grunfeld, E.A., Hunter, M.S., 2015. A systematic review of the factors associated with delays in medical and psychological help-seeking among men. Health Psychology Review 9, 264-76. 


\section{Click here to access/download Conflict of Interest Form McMahon et al PM Author Declaration.pdf}


Search strategy for CINAHL Plus

\section{Participants $(P)$}

S1. (MH "Men")

S2. ( MH "Male")

\section{Intervention (I)}

S3. ( MH "Telemedicine")

S4. (MH "Telehealth")

S5. (MH "Mobile applications") OR "mobile app*"

S6. ( MH "Smartphone")

S7. (MH "Cellular Phone") OR "cell phone"

S8. "Cellphone"

S9. "Mobile devices"

S10. (MH"Textmessaging")

S11. (MH"'Instantmessaging")

S12. "eHealth"

S13. "e-Health"

S14. "mHealth"

S15. "m-Health"

S16. "portable software"

S17. "online program"

\section{Outcomes (0)}

S18. (MH "Cardiovascular Diseases"

S19. (MH "Heart diseases")

S20. (MH "Exercise")

S21. (MH "Physical Activity") 
S22. "healthy diet"

S23. (MH "Nutrition")

S24. (MH "Smoking cessation")

S25. (MH "Cholesterol")

S26. (MH "Lipids")

S27.(MH"BloodPressure")

S28. (MH "Weight Control")

S29. (MH "Myocardial Ischemia")

S30. "coronary*"

S31. "Cardiac"

S32. (MH "Cardiovascular Risk Factors")

\section{Study design (S)}

S33. "Randomised controlled trial"

S34. "(MH "Randomized Controlled Trials")

S35. "RCT"

S36. (MH "Clinical Trials")

S37. S1 or S2

S38. S3ORS4ORS5ORS6ORS7ORS8ORS9ORS10ORS11ORS12ORS13ORS14ORS15ORS16 OR S17

S39. S18OR S19 OR S20 OR S21 OR S22 OR S23OR 24 OR S25OR S26 OR S27 OR S28OR S29 OR S30 OR S31 ORS32

S40. S33 OR S34 OR S35 OR S36

S41. S37 AND S38 AND S39 AND S40 
PRISMA 2009 Checklist

\begin{tabular}{|c|c|c|c|}
\hline Section/topic & \# & Checklist item & $\begin{array}{l}\text { Reported on } \\
\text { page \# }\end{array}$ \\
\hline \multicolumn{4}{|l|}{ TITLE } \\
\hline Title & 1 & Identify the report as a systematic review, meta-analysis, or both. & 1 \\
\hline \multicolumn{4}{|l|}{ ABSTRACT } \\
\hline Structured summary & 2 & $\begin{array}{l}\text { Provide a structured summary including, as applicable: background; objectives; data sources; study eligibility } \\
\text { criteria, participants, and interventions; study appraisal and synthesis methods; results; limitations; conclusions } \\
\text { and implications of key findings; systematic review registration number. }\end{array}$ & 2 \\
\hline \multicolumn{4}{|l|}{ INTRODUCTION } \\
\hline Rationale & 3 & Describe the rationale for the review in the context of what is already known. & $3-4$ \\
\hline Objectives & 4 & $\begin{array}{l}\text { Provide an explicit statement of questions being addressed with reference to participants, interventions, } \\
\text { comparisons, outcomes, and study design (PICOS). }\end{array}$ & 4 \\
\hline \multicolumn{4}{|l|}{ METHODS } \\
\hline Protocol and registration & 5 & $\begin{array}{l}\text { Indicate if a review protocol exists, if and where it can be accessed (e.g., Web address), and, if available, } \\
\text { provide registration information including registration number. }\end{array}$ & 4 \\
\hline Eligibility criteria & 6 & $\begin{array}{l}\text { Specify study characteristics (e.g., PICOS, length of follow-up) and report characteristics (e.g., years } \\
\text { considered, language, publication status) used as criteria for eligibility, giving rationale. }\end{array}$ & $4-5$ \\
\hline Information sources & 7 & $\begin{array}{l}\text { Describe all information sources (e.g., databases with dates of coverage, contact with study authors to identify } \\
\text { additional studies) in the search and date last searched. }\end{array}$ & 4 \\
\hline Search & 8 & $\begin{array}{l}\text { Present full electronic search strategy for at least one database, including any limits used, such that it could be } \\
\text { repeated. }\end{array}$ & $\begin{array}{l}\text { Supplementary } \\
\text { material (S1) }\end{array}$ \\
\hline Study selection & 9 & $\begin{array}{l}\text { State the process for selecting studies (i.e., screening, eligibility, included in systematic review, and, if } \\
\text { applicable, included in the meta-analysis). }\end{array}$ & 5 \\
\hline Data collection process & 10 & $\begin{array}{l}\text { Describe method of data extraction from reports (e.g., piloted forms, independently, in duplicate) and any } \\
\text { processes for obtaining and confirming data from investigators. }\end{array}$ & 5 \\
\hline Data items & 11 & $\begin{array}{l}\text { List and define all variables for which data were sought (e.g., PICOS, funding sources) and any assumptions } \\
\text { and simplifications made. }\end{array}$ & 5 \\
\hline $\begin{array}{l}\text { Risk of bias in individual } \\
\text { studies }\end{array}$ & 12 & $\begin{array}{l}\text { Describe methods used for assessing risk of bias of individual studies (including specification of whether this } \\
\text { was done at the study or outcome level), and how this information is to be used in any data synthesis. }\end{array}$ & 6 and Table 1 \\
\hline Summary measures & 13 & State the principal summary measures (e.g., risk ratio, difference in means). & $5-6$ \\
\hline Synthesis of results & 14 & $\begin{array}{l}\text { Describe the methods of handling data and combining results of studies, if done, including measures of } \\
\text { consistency }\left(e . g ., I^{2}\right) \text { for each meta-analysis. }\end{array}$ & 6 \\
\hline
\end{tabular}


PRISMA 2009 Checklist

Page 1 of 2

\begin{tabular}{|c|c|c|c|}
\hline Section/topic & $\#$ & Checklist item & $\begin{array}{l}\text { Reported } \\
\text { on page \# }\end{array}$ \\
\hline Risk of bias across studies & 15 & $\begin{array}{l}\text { Specify any assessment of risk of bias that may affect the cumulative evidence (e.g., publication bias, selective } \\
\text { reporting within studies). }\end{array}$ & 6 \\
\hline Additional analyses & 16 & $\begin{array}{l}\text { Describe methods of additional analyses (e.g., sensitivity or subgroup analyses, meta-regression), if done, indicating } \\
\text { which were pre-specified. }\end{array}$ & $5-6$ \\
\hline \multicolumn{4}{|l|}{ RESULTS } \\
\hline Study selection & 17 & $\begin{array}{l}\text { Give numbers of studies screened, assessed for eligibility, and included in the review, with reasons for exclusions at } \\
\text { each stage, ideally with a flow diagram. }\end{array}$ & $\begin{array}{l}\text { 6-7 and } \\
\text { Figure } 1\end{array}$ \\
\hline Study characteristics & 18 & $\begin{array}{l}\text { For each study, present characteristics for which data were extracted (e.g., study size, PICOS, follow-up period) and } \\
\text { provide the citations. }\end{array}$ & $\begin{array}{l}\text { 8-9 and } \\
\text { Table } 2 \\
\end{array}$ \\
\hline Risk of bias within studies & 19 & Present data on risk of bias of each study and, if available, any outcome level assessment (see item 12). & $9-11$ \\
\hline Results of individual studies & 20 & $\begin{array}{l}\text { For all outcomes considered (benefits or harms), present, for each study: (a) simple summary data for each } \\
\text { intervention group (b) effect estimates and confidence intervals, ideally with a forest plot. }\end{array}$ & $\begin{array}{l}11-12, \\
18-19 \\
\text { and } \\
\text { Figures } \\
3-7\end{array}$ \\
\hline Synthesis of results & 21 & Present results of each meta-analysis done, including confidence intervals and measures of consistency. & $\begin{array}{l}\text { 11-12, } \\
18-19 \\
\text { and } \\
\text { Figures } \\
3-7\end{array}$ \\
\hline Risk of bias across studies & 22 & Present results of any assessment of risk of bias across studies (see Item 15). & $\begin{array}{l}\text { 8-11 and } \\
\text { Figure } 2\end{array}$ \\
\hline Additional analysis & 23 & Give results of additional analyses, if done (e.g., sensitivity or subgroup analyses, meta-regression [see Item 16]). & $\begin{array}{l}11-12 \\
18-19\end{array}$ \\
\hline \multicolumn{4}{|l|}{ DISCUSSION } \\
\hline Summary of evidence & 24 & $\begin{array}{l}\text { Summarize the main findings including the strength of evidence for each main outcome; consider their relevance to } \\
\text { key groups (e.g., healthcare providers, users, and policy makers). }\end{array}$ & $22-24$ \\
\hline Limitations & 25 & $\begin{array}{l}\text { Discuss limitations at study and outcome level (e.g., risk of bias), and at review-level (e.g., incomplete retrieval of } \\
\text { identified research, reporting bias). }\end{array}$ & 24 \\
\hline
\end{tabular}


PRISMA 2009 Checklist

\begin{tabular}{|l|r|l|l|}
\hline Conclusions & 26 & Provide a general interpretation of the results in the context of other evidence, and implications for future research. & 24 \\
\hline FUNDING & 27 & $\begin{array}{l}\text { Describe sources of funding for the systematic review and other support (e.g., supply of data); role of funders for the } \\
\text { systematic review. }\end{array}$ & 24 \\
\hline Funding
\end{tabular}

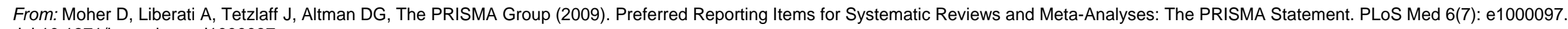
doi:10.1371/journal.pmed1000097

For more information, visit: www.prisma-statement.org

Page 2 of 2 